\title{
Histological Detection of Minimal Metastatic Involvement in Axillary Sentinel Nodes: A Rational Basis for a Sensitive Methodology Usable in Daily Practice
}

Paul Fréneaux, M.D., Claude Nos, M.D., Anne Vincent-Salomon, M.D., Pascal Genin, M.D., Brigitte Sigal-Zafrani, M.D., Abir Al Ghuzlan, M.D., Marie-José Birolini, M.D., Krishna Clough, M.D., Xavier Sastre-Garau, M.D.

Departments of Pathology (PF, AV-S, PG, BS-Z, AAG, M-JB, XS-G) and Surgery (CN, KC), Institut Curie, Paris, France

There is no consensus method for the histological analysis of axillary sentinel nodes (SN). This study aimed to (1) assess the rate of occult metastases in SN using large serial sectioning and immunohistochemistry (IHC), (2) evaluate whether occult metastases were predictive of metastases in the downstream axillary nodes, and (3) specify a methodology of analysis of $\mathrm{SN}$ that could be both sensitive and applicable in daily practice. One hundred three patients with breast carcinoma underwent $\mathrm{SN}$ biopsy and then axillary dissection. SN free of tumor at standard examination of one section were sectioned at six levels (150- $\mu \mathrm{m}$ intervals) and immunostained for cytokeratin. The number and localization of labeled metastatic cells (occult metastases) were recorded. In 29 of the 103 patients $(28 \%)$, SN were found to be metastatic after standard examination. The SN of the remaining 74 patients were further analyzed using IHC. Occult metastases were detected in 35 of these patients $(47.3 \%)$, leading to an overall SN involvement rate of $62 \%(29+35 / 103)$. In 33 of these 35 cases, the plurality and the dispersion of the immunostained cells implied that the screening of only 3 of the 6 levels would have led to the detection of diagnostic positive events. Only one of the 35 patients $(2.8 \%)$ with occult metastases showed metastatic lymph node in the downstream axilla. In our series of axillary $\mathrm{SN}$, the analysis of one standard histologic section and, when negative, of only three additional sections after IHC revealed $>\mathbf{6 0 \%}$ of metastasis or occult metastasis. Metastasis detected by standard analysis had a high predictive

Copyright (C) 2002 by The United States and Canadian Academy of Pathology, Inc.

VOL. 15, NO. 6, P. 641, 2002 Printed in the U.S.A.

Date of acceptance: March 7, 2002.

Address reprint requests to: Xavier Sastre-Garau, M.D., Service de $\mathrm{Pa}$ thologie, Institut Curie, 26 rue d'Ulm, 75248 Paris Cedex 05, France; e-mail: xavier.sastre@curie.net; fax: +01 44324072. value of downstream node metastasis, whereas the predictive value of occult metastasis revealed by IHC was poor. The clinical significance of occult metastases in $\mathrm{SN}$ needs to be specified by long-term follow-up analysis.

KEY WORDS: Blue dye, Breast carcinoma, Immunohistochemistry, Micrometastases, Occult metastases, Sentinel lymph nodes, Serial sections.

Mod Pathol 2002;15(6):641-646

Morbidity related to sentinel node (SN) surgery is lower than that for lymphadenectomy (1). SN biopsy is thus an advantageous alternative to axillary lymph nodes dissection in the staging of breast carcinoma (2-5). When $\mathrm{SN}$ is metastatic, axillary lymphadenectomy is indicated. However, there is no standardized procedure for the histopathological analysis of SN. The significance of the detection of minimal metastatic involvement and the methodology to be used for this detection must be specified (6-9). Lymph node metastatic deposits sized $<2 \mathrm{~mm}$ are called micro-metastases (according to the International Union Against Cancer; UICC), whereas occult metastases correspond to minimal involvement revealed only by immunohistochemistry (IHC; 10).

The frequency of occult metastasis found in axillary lymph nodes is proportional to the number of histological sections analyzed $(8,11-13)$. IHC increases this frequency $(8,13-18)$. However, this technique is time consuming, and its interest is limited in the histological assessment of lymphadenectomy because the prognostic value of minimal lymph node involvement is low (13). However, such a procedure may be indicated in the analysis of $\mathrm{SN}$ : a minimal metastatic involvement of a $\mathrm{SN}$ may be associated with the metastatic extension of one or of several downstream lymphatic nodes $(19,20)$. 
Moreover, the prognostic value of occult metastasis in $\mathrm{SN}$ has to be determined. This problem prompted us to perform an extensive analysis of SN, using serial histologic sections and IHC, in a series of 103 patients. In all cases, axillary lymphadenectomy was also performed and lymph nodes were analyzed separately. Our aims were (1) to assess the rate of metastasis and of occult metastasis in our series of SN, (2) to compare the levels of metastatic involvement in the $\mathrm{SN}$ and in the downstream nodes for each patient (3), to determine which methodology presenting an optimal sensitivity could be acceptable in daily practice, and (4) to standardize the methodology of histological analysis of SN, to assess, over the long term, the prognostic value of minimal metastatic disease in SN.

\section{MATERIALS AND METHODS}

From December 1997 to December 1998, 103 patients treated at the Institut Curie for a breast carcinoma underwent SN biopsy, immediately followed by standard axillary dissection. Patients and tumor characteristics (histological type, histoprognostic grade, and hormonal status) are listed in Table 1 . The mean age of patients was 58 years (range, 29 to $87 \mathrm{y}$ ). All tumors corresponded to invasive carcinoma. The first therapeutic procedure was lumpectomy (89\% of the cases) or mastectomy (11\%). The mean tumor size was $17.4 \mathrm{~mm}$ (range, 5 to $50 \mathrm{~mm}$ ). Blue dye was used to identify the SN in all cases. The dye (2 to $5 \mathrm{~mL}$ ) was injected into the breast tumor mass and in the surrounding tissue, in the direction of the axilla, not into the dermis or into the hypodermis. In 55 cases (53\%), the tumor had been excised in a first surgical step. In these cases, the blue dye was injected in the wall of the biopsy cavity and in the surrounding tissue. After injection of the dye, the mammary gland was massaged for 10 to 20 minutes. A separate incision in the axilla was then performed to localize a bluestained lymphatic vessel, which was followed to the stained SN. This node was excised, then a standard axillary dissection including Level I and II lymphatic nodes was performed.

All lymph nodes (sentinels or not sentinels) were identified separately and fixed in AFA (5\% acetic acid, $75 \%$ pure ethanol, $18 \%$ demineralized water, and $2 \%$ formalin). They were cut in two, three, or four parts of equal thickness. Nodes of $<0.3 \mathrm{~cm}$ were not cut. Each lymph node was separately embedded in paraffin. One $3-\mu \mathrm{m}$-thick section was removed from each block and stained with HES (hematein, eosin, saffron).

Cases with all SN free of metastasis or of micrometastasis $(<2 \mathrm{~mm}$; UICC) after standard microscopic examination were further analyzed using IHC to disclose occult metastasis (10). Considering the mean size of an epithelial cell (about $20 \mu \mathrm{m}$ ), serial scanning at $150-\mu \mathrm{m}$ intervals looked to be rational for revealing any clusters of 5 to 10 malignant cells. On each block, six serial histologic sections taken at $150-\mu \mathrm{m}$ intervals were prepared (Fig. 1). According to this procedure, each $\mathrm{SN}$ was scanned on at least $0.9 \mathrm{~mm}$ thick. Larger lymph nodes cut into two, three, or four slices were scanned on a thickness of $\geq 1.8,2.7$, or $3.6 \mathrm{~mm}$, respectively. The sections analyzed by IHC were numbered from 1 to 6 from the equator (Fig. 1). After microscopic examination, the number and the type (isolated cells or clusters) of stained cells was registered. Morphological analysis allowed exclu-

TABLE 1. Patient and Tumor Characteristics According to Sentinel Node Status

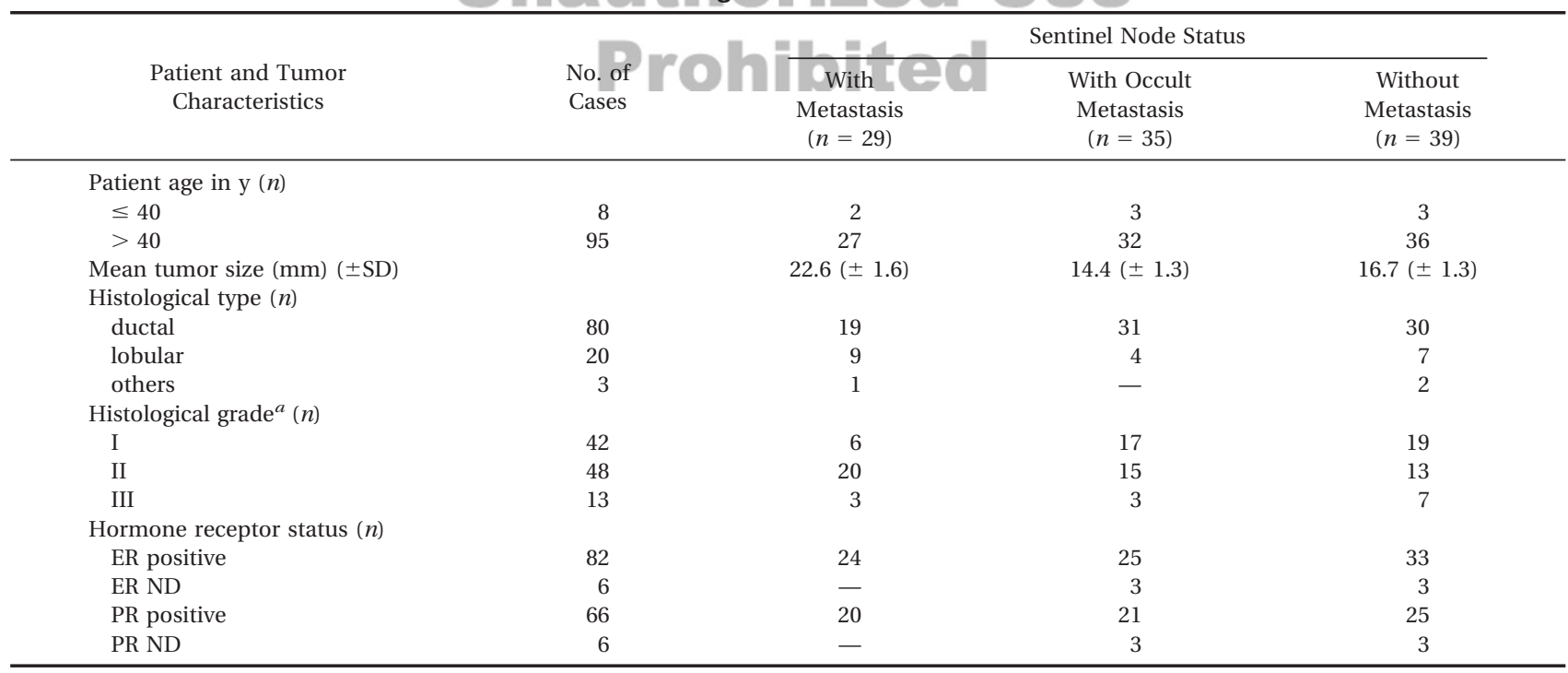

$\mathrm{ND}$, not done; ER, estrogen receptor; PR, progesterone receptor.

${ }^{a}$ Nottingham index. 


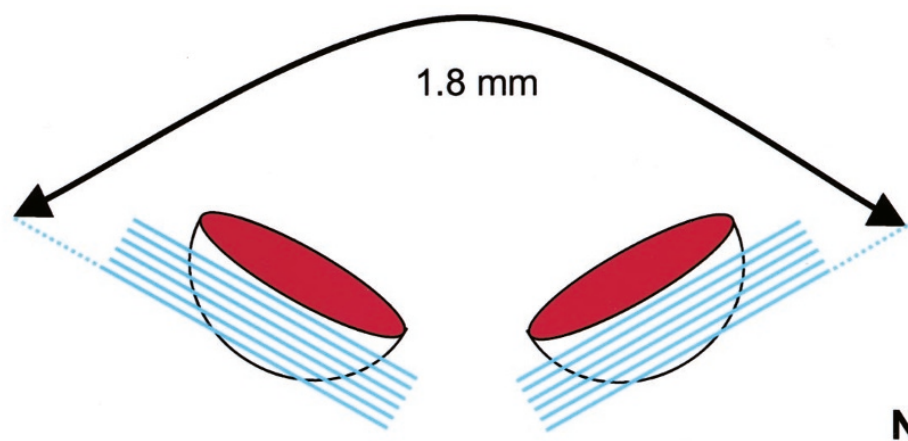

Number of slides with immunostained cells

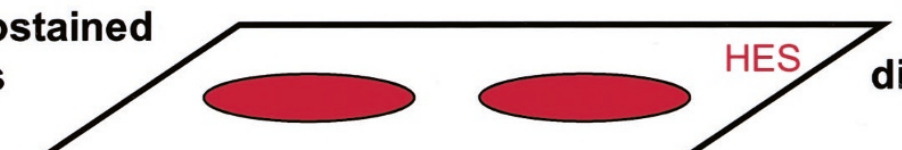

Number of new positive cases successively disclosed at each level

23/149

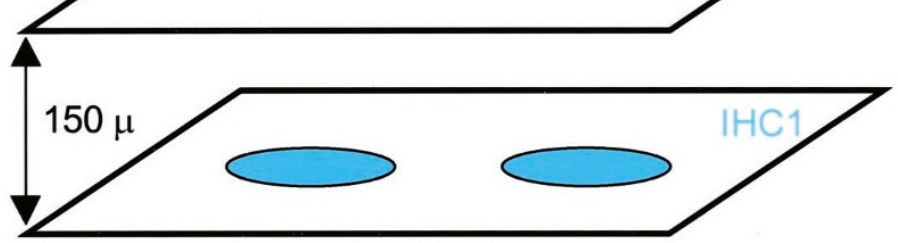

$20 / 74$

$22 / 149$

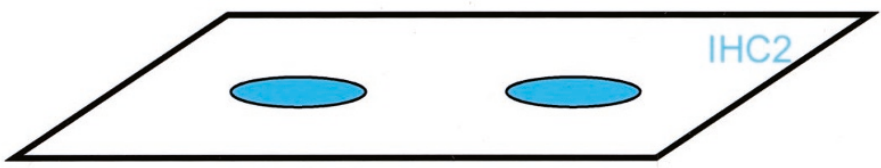

$7 / 74$

23/149

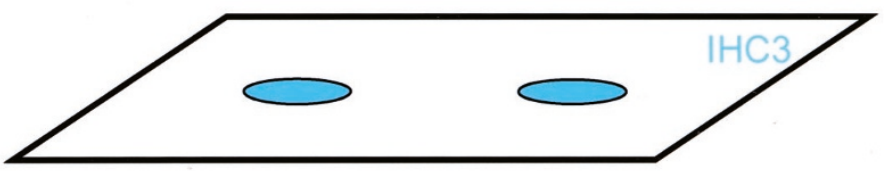

$6 / 74$

$17 / 149$

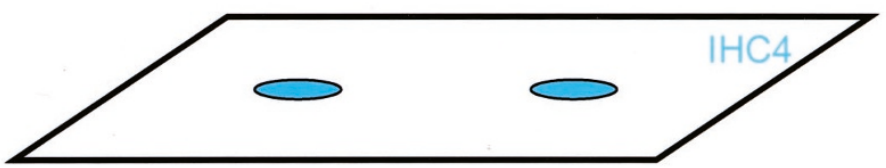

$1 / 74$

$15 / 149$

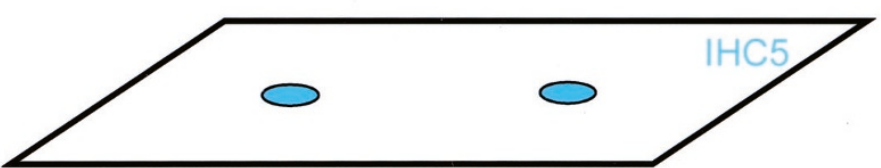

$0 / 74$

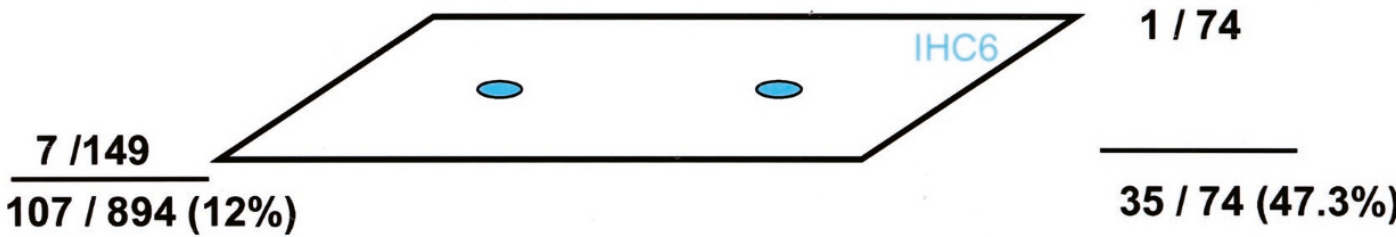

FIGURE 1. Methodology and results of the analysis of the SN categorized by slides (left column) and by cases (right column).

sion of irrelevant staining of macrophages or of endothelial cells in some cases. Only cells with morphology compatible with that of epithelial cells and showing unambiguous cytoplasmic labeling were considered positive events.
IHC was performed using pan-anti-cytokeratin antibody (Kl1, Immunotech, Marseille, France). After rehydration and antigen retrieval in citrate buffer (10 mM, pH 6.1), tissue sections were incubated with the primary monoclonal antibody, for 1 
hour, at 1/100 dilution. The revelation of the staining was performed using the Vectastain Elite $A B C$ peroxidase mouse IgG kit (Vector Burlingame, CA) and the diamino-benzidine (DAKO A/S, Glostrup, Denmark), as chromogen. Immunoreactions were done with an automatic immunostainer (TechMate Horizon, LJL Biosystems Inc.).

\section{RESULTS}

This study concerns a series of 103 patients who underwent SN biopsy (214 SN; mean, 2 SN per patient; range, 1 to $6 \mathrm{SNs})$. After standard microscopic examination, metastatic SN were detected in 29 of these patients (28\%). In the remaining 74 cases (149 SN), the SN were further analyzed using IHC on six sections (894 immunostainings). After microscopic analysis, 107 of the 894 sections (12.2\%), corresponding to $43 \mathrm{SN}$, were found to harbor immunostained cells (Fig. 1). These slides with immunostained cells concerned 35 of the 74 patients (47.3\%; Fig. 1, right column). Preparations with stained cells were homogeneously distributed on the respective levels analyzed: 23 preparations with positive events corresponded to a Level 1,22 to a Level 2, 23 to a Level 3, 17 to a Level 4, 15 to a Level 5, and 7 to a Level 6 (Fig. 1, left column).

The assessment of the number of immunostained cells in the 35 cases with occult metastases showed that at least a cluster of stained cells (Fig. $2 \mathrm{~A}$ ) was found in 28 patients. Two of the 7 remaining cases exhibited 15 and 10 cells, respectively (Fig. 2B), whereas in each of the five last cases, only one cell was observed. It is of practical importance to stress that in 33/35 cases (95\%), the screening of the first three levels would have been sufficient for the detection of diagnostic positive events (Fig. 1, right column). In each of these 33 cases, the presence of immunostained cells on Levels 4 to 6 was accompanied by positive events on any of the first three levels. A similar rate of occult metastases was found by the analysis of any combination of three of the six immunostainings performed (data not shown).

The comparison of SN status and tumor characteristics revealed a positive correlation between tumor size and the presence of metastasis in $\mathrm{SN}(P<$ .001). No other significant correlation was found, although the rate of lobular carcinoma was higher in the group of tumors with metastatic SN than in the overall population.

The second step of our analysis was to compare the status of the SN with that of the axillary clearance. In 15 of the 29 cases with metastatic SN at standard staining $(51.7 \%)$, at least one lymph node was metastatic in the axillary lymphadenectomy (Table 2). Among the 35 cases with occult metastases detected by IHC, only one $(2.8 \%)$ showed metastatic lymph node in the axilla (Table 2). Conversely, in two of the 39 cases with no involvement of the SN after both standard and immunohistochemical analysis, one metastatic lymph node was found in the axillary clearance.

\section{DISCUSSION}

In this study, axillary SN were found to be metastatic at standard examination in $28 \%$ of the cases. In $47.3 \%$ of the patients with no metastatic SN, occult metastases were found using serial sectioning and IHC. This raises to $62 \%$ the overall rate of metastatic involvement in axillary SN. In the literature, the rate of metastasis and of occult metastasis in SN varies from 35 to $58 \%$ (Table 3). The high rate of $\mathrm{SN}$ involvement in our cases is likely to be related both to the large size of the corresponding breast tumors and to the extensive serial sectioning performed for the histopathological analysis. The disparity of the rates of SN involvement reported so far is largely due to the heterogeneity in the methods used for the analysis. For instance, the number of sections performed on each SN ranged from one (4) to $\geq 30(7,21)$. IHC significantly increases the
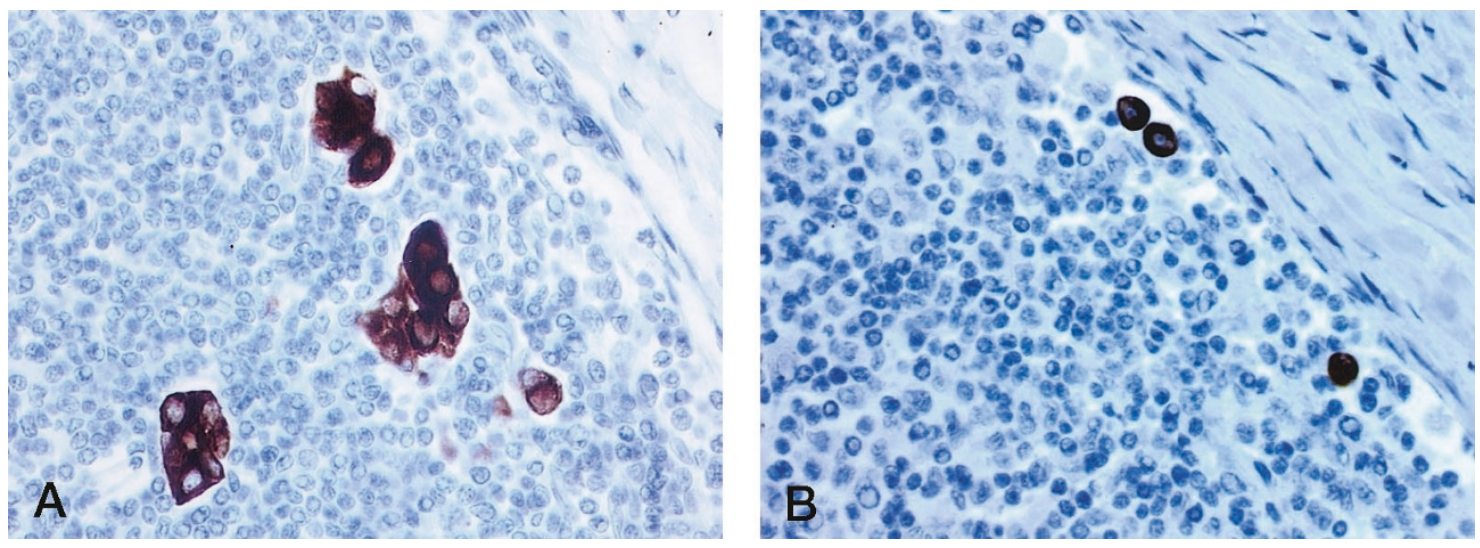

FIGURE 2. Occult metastases in axillary sentinel node revealed by immunohistochemistry: clusters of immunostained cells (A) and uncohesive immunostained cells (B). 
TABLE 2. Comparison between Results of Analysis of Sentinel Nodes and of Corresponding Axillary Clearance

\begin{tabular}{|c|c|c|c|}
\hline \multirow[b]{2}{*}{ Sentinel Node } & \multirow{2}{*}{$\begin{array}{l}\text { No. of } \\
\text { Cases }\end{array}$} & \multicolumn{2}{|c|}{ Corresponding Axillary Clearance } \\
\hline & & $\begin{array}{c}\text { With } \\
\text { Metastasis }\end{array}$ & $\begin{array}{c}\text { Without } \\
\text { Metastasis }\end{array}$ \\
\hline Metastatic at standard HES & 29 & $15(51.7 \%)$ & $14(48.3 \%)$ \\
\hline Metastatic at IHC & 35 & $1^{a}(2.8 \%)$ & $34(97.2 \%)$ \\
\hline Not metastatic & 39 & $2^{a}(5.1 \%)$ & $37(94.9 \%)$ \\
\hline Total & 103 & $18(17.5 \%)$ & $85(82.5 \%)$ \\
\hline
\end{tabular}

IHC, immunohistochemistry; HES, hematein, eosin, saffron.

${ }^{a}$ Only one metastatic lymph node.

frequency of metastatic involvement by revealing occult malignant cells independently dispersed or arranged in very small clusters (7). The $62 \%$ rate of $\mathrm{SN}$ involvement found in our series is not significantly different from the $58 \%$ rate reported after complete sectioning and scanning of the SN (7). The comparison with our data strongly suggests that over a threshold, the analysis of additional sections increases the number of stained cells detected but, in the large majority of cases, does not afford diagnostic information for individual patient. In our work, the repartition of the different metastatic events in each case had been recorded, and this analysis showed that a threshold was obtained at the rate of three histological sections performed every $150 \mu \mathrm{m}$. This corresponds to the scanning of about $0.9-\mathrm{mm}$ thickness of an SN included in two fragments. This sectioning might be lowered by increasing the number of macroscopic sections of the lymph nodes (15). It is also important to stress that the high frequency of occult metastases detected after IHC on serial sections is more often related to the increase in the surface of the node parenchyma analyzed than to IHC itself, which reveals very small aggregates or isolated epithelial cells. In our series, clusters of occult metastatic cells were found in 28 of the 35 cases that were labeled as positive after IHC-only analysis. Some of these clus- ters might have been detected by the analysis of these serial sections merely stained by HES. Consequently, our current protocol of analysis of axillary $\mathrm{SN}$ is now to perform one median histological section, plus three pairs of sections taken every 150 $\mu \mathrm{m}$. The median section and one of each couple of sections are analyzed after standard HES staining. When no micrometastasis are observed, IHC is performed on the three remaining sections to detect occult metastases. This procedure reduces the amount of immunostaining to be performed.

The second aspect in this study was the comparison between the status of SN and that of the corresponding axillary clearance. When the SN was metastatic at standard microscopic examination, metastasis in the downstream axillary node(s) was observed in $>50 \%$ of the cases. In contrast, when only occult metastasis were found in the SN, metastatic node was found in only one $(2.8 \%)$ case in the axilla. This rate is in the 0 to $5 \%$ range reported in the literature $(2-4,19,22)$ and shows that occult metastases have no predictive value of metastatic involvement of the downstream axilla. IHC on serial sectioning in axillary SN is thus of no interest for individual patients at the time of primary treatment and should not influence clinical decision making $(6,9)$. The prognostic significance of micrometastasis and of occult metastasis in SN is unknown. In axillary clearance, the prognostic value of minimal metastatic involvement has been diversely assessed $(8,13)$. Recent works, including a large number of patients and a long follow-up, report a lower rate of disease-free survival in patients when occult metastases are found in axillary nodes (13-17). However, the incidence of occult metastases in disease outcome is also dependent on other factors, such as the histological type of tumors (15) or the menopausal status of patients (17). The individualization of axillary SN provides the opportunity for an accu-

TABLE 3. Results of Sentinel Lymph Node Analysis in the Literature

\begin{tabular}{|c|c|c|c|c|c|c|}
\hline Author & Year & $\begin{array}{l}\text { No. of } \\
\text { cases }\end{array}$ & $\begin{array}{l}\text { No. of sections } \\
\text { (HES/IHC) }\end{array}$ & $\begin{array}{c}\text { SN+/HES } \\
\text { No }(\%)\end{array}$ & $\begin{array}{l}\mathrm{SN}+/ \mathrm{IHC} \\
\text { No }(\%)\end{array}$ & $\begin{array}{c}\text { Overall SN+ } \\
\text { No (\%) }\end{array}$ \\
\hline Giuliano et al. (23) & 1995 & 162 & $1 / 3-4$ & 57 (35.2) & $11(6.8)$ & $68(42)$ \\
\hline Giuliano et al. (19) & 1997 & 100 & $2 / 1$ & $33(33)$ & $9(9)$ & $42(42)$ \\
\hline Turner et al. (2) & 1997 & 103 & $2 / 1$ & $33(32)$ & $10(10)$ & $43(42)$ \\
\hline Turner et al. (18) & 1999 & 52 & $2 / 10$ & $10(19.2)$ & $9(17.3)$ & $19(36.5)$ \\
\hline Turner et al. (24) & 2000 & 514 & $1-3 / 1$ & $152(29.6)$ & $62(12)$ & $214(41.6)$ \\
\hline Veronesi et al. (3) & 1997 & 160 & $3 / 0$ & $81(50.6)$ & - & $81(50.6)$ \\
\hline \multirow[t]{3}{*}{ Veronesi et al. (21) } & 1999 & 60 & $1 / 0$ & $35(58.3)$ & - & 35 (58.3) \\
\hline & & 192 & $4 / 0$ & $81(42.2)$ & - & $81(42.2)$ \\
\hline & & 119 & $15 / 15$ & NS & NS & $52(43.7)$ \\
\hline Krag et al. (4) & 1998 & 405 & $1 / 0$ & $101(25)$ & - & $101(25)$ \\
\hline Offodile et al. (20) & 1998 & 41 & $1 / 1$ & $15(36.6)$ & $3(7.3)$ & $18(44)$ \\
\hline Dowlatshahi et al. (7) & 1999 & 52 & $1 / \mathrm{n}^{a}$ & $6(11.6)$ & $24(46.2)$ & $30(58)$ \\
\hline Doting et al. (25) & 2000 & 126 & $3 / 3$ & NS & NS & $56(44)$ \\
\hline Torrenga et al. (26) & 2001 & 250 & $5 / 5$ & $76(30.4)$ & $10(4)$ & $86(34.4)$ \\
\hline Fréneaux et al. (this study) & 2001 & 103 & $1 / 6$ & $29(28)$ & $35(34)$ & $64(62)$ \\
\hline
\end{tabular}

NS, not specified; IHC, immunohistochemistry; HES, hematein, eosin, saffron; SN, sentinel node(s).

${ }^{a}$ Serial sections of the entire node. 
rate assessment of the prognostic value of minimal lymph node involvement in breast cancers.

In our analysis, occult metastases were found on 107 immunostained slides, corresponding to 43 SN removed from 35 patients. There was thus a mean of three slides with occult metastases per patient, prepared, in most of the cases, from only one of the two SN isolated in each of these patients. It is important to underline that when no occult metastases are found in $\mathrm{SN}$, downstream axillary nodes are generally free of occult metastases (22). The parameters that condition the elective involvement of only one SN are unclear. Malignant epithelial cells may be temporarily arrested in the first relay of the immune system. Alternatively, the hypothesis of a co-migration of the dye and of the epithelial cells has not been excluded.

In conclusion, our study and data from the literature indicate that metastases in $\mathrm{SN}$ detected by standard histological analysis have strong predictive value for the involvement of downstream axillary nodes. Serial sectioning enhances the number of minimal metastatic deposits found in SN. However, an analysis limited to three histological sections taken at $150-\mu \mathrm{m}$ intervals provides the diagnosis for the majority of patients, and such a methodology is easily applicable in daily practice. Occult metastases, detected by IHC only, have no predictive value of the status of the downstream axilla. Axillary dissection should not be performed only secondarily to the detection of immunostained cells. The prognostic value of occult metastasis in SN will be assessed by a long-term follow-up study including a large number of patients. From this perspective, it is important to standardize the technique of the histopathological analysis of $\mathrm{SN}$ and to record in pathological reports whether SN involvement corresponds to a metastasis, micrometastasis, or occult metastasis, the respective biological value of which are likely to be different.

\section{REFERENCES}

1. Giuliano A, Kirgan D, Guenther J, Morton D. Lymphatic mapping and sentinel lymphadenectomy for breast cancer. Ann Surg 1994;220:391-401.

2. Turner R, Ollila D, Krasne D, Giuliano A. Histopathologic validation of the sentinel lymph node hypothesis for breast carcinoma. Ann Surg 1997;226:271-8.

3. Veronesi U, Paganelli G, Galimberti V, Viale G, Zurrida S, Bedoni M, et al. Sentinel-node biopsy to avoid axillary dissection in breast cancer with clinically negative lymph nodes. Lancet 1997;349:1864-7.

4. Krag D, Weaver D, Ashikaga T, Moffat F, Klimberg V, Shriver $\mathrm{C}$, et al. The sentinel node in breast cancer. A multicenter validation study. N Engl J Med 1998;339:941-6.

5. O'Hea B, Hill A, El-Shirbiny A, Yeh S, Rosen P, Coit D, et al. Sentinel lymph node biopsy in breast cancer: initial experience at Memorial Sloan-Kettering Cancer Center. J Am Coll Surg 1998;186:423-7.
6. McIntosh S, Going J, Soukop M, Purushotham A, Cooke T. Therapeutic implications of the sentinel lymph node in breast cancer. Lancet 1999;354:570.

7. Dowlatshahi K, Fan M, Bloom K, Spitz D, Patel S, Snider H. Occult metastases in the sentinel lymph nodes of patients with early stage breast carcinoma. A preliminary study. Cancer 1999;86:990-6.

8. Dowlatshahi K, Fan M, Snider H, Habib F. Lymph node micrometastases from breast carcinoma. Reviewing the dilemma. Cancer 1997;80:1188-97.

9. Allred D, Elledge R. Caution concerning micrometastatic breast carcinoma in sentinel lymph nodes. Cancer 1999;86: 905-7.

10. Hermanek P, Hutter R, Sobin L, Wittekind C. International Union Against Cancer: classification of isolated tumor cells and micrometastasis. Cancer 1999;86:2668-73.

11. International (Ludwig) Breast Cancer Study Group. Prognostic importance of occult axillary lymph node micrometastases from breast cancers. Lancet 1990;335:1565-8.

12. Neville A, Price K, Gelber R, Goldhirsch A. Axillary node micrometastases and breast cancer. Lancet 1991;337:1110.

13. Nasser I, Lee A, Bosari S, Saganich R, Heatley G, Silverman M. Occult axillary lymph node metastases in "nodenegative" breast carcinoma. Hum Pathol 1993;24:950-7.

14. Trojani M, de Mascarel I, Bonichon F, Coindre J, Delsol G. Micrometastases to axillary lymph nodes from carcinoma of the breast. Br J Cancer 1987;55:303-6.

15. de Mascarel I, Bonichon F, Coindre J, Trojani M. Prognostic significance of breast cancer axillary node micrometastases assessed by two special techniques: re-evaluation with longer follow-up. Br J Cancer 1992;66:523-7.

16. McGuckin M, Cummings M, Walsh M, Hohn B, Bennett I, Wright R. Occult axillary node metastases in breast cancer: their detection and prognostic significance. $\mathrm{Br} \mathrm{J}$ Cancer 1996;73:88-95.

17. Cote R, Peterson H, Chaiwun B, Gelber R, Goldhirsch A, Castiglione-Gertsch $\mathrm{M}$, et al. Role of immunohistochemical detection of lymph-node metastases in management of breast cancer. Lancet 1999;354:896-900.

18. Turner R, Ollila D, Stern S, Giuliano A. Optimal histopathologic examination of the sentinel lymph node for breast carcinoma staging. Am J Surg Pathol 1999;23:263-7.

19. Giuliano A, Jones R, Brennan M, Statman R. Sentinel lymphadenectomy in breast cancer. J Clin Oncol 1997;15:2345-50.

20. Offodile R, Hoh C, Barsky S, Nelson S, Elashoff R, Eilber F, et al. Minimally invasive breast carcinoma staging using lymphatic mapping with radiolabeled dextran. Cancer 1998;82:1704-8.

21. Veronesi U, Paganelli G, Viale G, Galimberti V, Luini A, Zurrida S, et al. Sentinel lymph node biopsy and axillary dissection in breast cancer: results in a large series. J Natl Cancer Inst 1999;91:368-73.

22. Sabel M, Zhang P, Barnwell J, Winston J, Hurd T, Edge S. Accuracy of sentinel node biopsy in predicting nodal status in patients with breast carcinoma. J Surg Oncol 2001;77:243-6.

23. Giuliano A, Dale P, Turner R, Morton D, Evans S, Krasne D. Improved axillary staging of breast cancer with sentinel lymphadenectomy. Annu Surg 1995;222:394-401.

24. Turner R, Chu K, Qi K, Botnick L, Hansen N, Glass E, et al. Pathologic features associated with nonsentinel lymph node metastases in patients with metastatic breast carcinoma in a sentinel lymph node. Cancer 2000;89:574-81.

25. Doting M, Jansen L, Nieweg O, Piers D, Tiebosch A, Schraffordt Koops $\mathrm{H}$, et al. Lymphatic mapping with intralesional tracer administration in breast carcinoma patients. Cancer 2000;88:2546-52.

26. Torrenga H, Rahusen F, Meijer S, Borgstein P, Van Diest P. Sentinel node investigation in breast cancer: detailed analysis of the yield from step sectioning and immunohistochemistry. J Clin Pathol 2001;54:550-2. 